\title{
Frequency Response of Strain-Compensated InGaAsN-GaAsP-GaAs SQW Lasers
}

\author{
Ovidio H. Anton, Student Member, IEEE, Dinesh Patel, Carmen S. Menoni, Senior Member, IEEE, Jeng-Ya Yeh, \\ T. T. Van Roy, L. J. Mawst, Senior Member, IEEE, J. M. Pikal, Member, IEEE, and Nelson Tansu
}

\begin{abstract}
We investigate the below and above threshold frequency response of InGaAsN lasers with different nitrogen content. This is accomplished through detailed analysis of the small signal modulation response of the laser diodes using a comprehensive model based on rate equations and that incorporates the effect of parasitics. For below threshold conditions the model is instrumental in separating the contributions from the parasitics (more severe at low bias) and carrier recombination (predominant at higher bias) to the measured carrier lifetime. It is found that the addition of nitrogen reduces the recombination lifetime, mainly as a result of a four-fold increase in monomolecular recombination which predominates even near threshold. For bias above threshold the analysis compares electrical versus optical modulation frequency responses and concludes that resonance frequency and damping extracted from the electrical modulation responses are significantly influenced by the device parasitics. Instead, it is shown that optical modulation traces allow extraction of a relaxation frequency that is shaped only by the stimulated processes in the laser active region. Even in this case, the damping is found to be affected by the parasitics. When compared with nitrogen free lasers, a reduction in the resonance frequency is observed, while the damping is not altered. The former arises from a factor of $\sim 2.5$ decrease in the combined effect of the differential gain and carrier transport parameters. The latter reflects the more significant contribution of the parasitics to the damping.
\end{abstract}

Index Terms-Dilute nitride quantum-well (QW) lasers, frequency response: modeling and experiment.

\section{INTRODUCTION}

A NALYSIS OF below and above threshold frequency responses has been instrumental to unravel intrinsic properties that shape the output characteristics of laser diodes. Below threshold, these investigations allow one to obtain the carrier recombination rate, and from its behavior with bias assess the dominant recombination processes taking place in the laser active region [1]. These investigations in InP based laser diodes

Manuscript received December 1, 2004; revised June 15, 2005. This work was supported by the National Science Foundation under Grant ECS 03134410. The work of O. Anton was supported in part by the Agilent Photonics Fellowship, Agilent Technologies, Palo Alto, CA. The work of J. M. Pikal was supported by the Army Research Office (ARO) under Contract DAAD19-01-1-0567.

O. H. Anton, D. Patel, and C. S. Menoni are with the Department of Electrical and Computer Engineering, Colorado State University, Fort Collins, CO 805231373 USA (e-mail: anton@engr.colostate.edu; carmen@engr.colostate.edu).

J.-Y. Yeh, T. T. Van Roy, and L. J. Mawst are with the Reed Center for Photonics, Department of Electrical and Computer Engineering, University of Wisconsin-Madison, Madison, WI 53706 USA.

J. M. Pikal is with the Department of Electrical and Computer Engineering, University of Wyoming, Laramie, WY 82071 USA.

$\mathrm{N}$. Tansu is with the Center for Optical Technologies, Department of Electrical and Computer Engineering, Lehigh University, Bethlehem, PA 18015 USA.

Digital Object Identifier 10.1109/JSTQE.2005.853845 have elucidated the important role of Auger recombination in the output characteristics of these devices [2], [3]. Above threshold, frequency response measurements assess the modulation bandwidth of the devices, while at the same time allowing investigation of the device and material's processes that shape the high frequency behavior [4]-[10].

The recent demonstration of $1.3-\mu \mathrm{m}$ laser diodes based on the incorporation of nitrogen in InGaAs, with emission wavelengths in the important telecom range $(1.3-1.5 \mu \mathrm{m})$ [11]-[18], opens new grounds for investigating the impact of the material's physics and device structure on the dynamic behavior of the lasers. Specially since the mechanism by which the long wavelength emission is realized involves the repulsive interaction of the host InGaAs conduction band states and nitrogen impuritylike states [19], [20]. Most of the experimental work on the dynamic response of dilute nitride laser diodes reported so far has benchmarked the laser bandwidth [21]-[23]. The results of these experiments are very encouraging as they show the dilute nitride lasers have potential to realize modulation bandwidths comparable to their InP counterparts [22], [24]. There are not, to our knowledge, experimental studies that investigate in depth the physical mechanisms that affect the carrier dynamics in the dilute nitride lasers. These investigations, until now restricted to theoretical work, have provided insight that monomolecular recombination significantly increases with nitrogen incorporation in the strained InGaAs wells, thereby reducing the carrier lifetime [25]. Model calculations also reveal a significant reduction in the peak gain and differential gain with increased nitrogen content in the laser active region [26]-[28]. Both of these parameters affect the above threshold high frequency response. Testing of the theoretical predictions against experiments is not trivial, particularly in broad area (BA) and ridge waveguide (RWG) InGaAsN diodes where the external circuitry and device parasitics contribute to the modulation response.

Herein, we describe a comprehensive analysis of the below and above threshold modulation response of InGaAsN lasers diodes. The analysis encompasses a simulation based on rate equations that contemplate the effect of parasitics complemented by the experiments of the below and above threshold modulation responses. The experiments are carried out in two identical structures which only differ in the nitrogen content in the well. Their results are analyzed with the model to assess the impact of nitrogen incorporation on the intrinsic carrier dynamics behavior. The model is essential to reveal the impact of monomolecular recombination in shaping the dynamics of carrier recombination even at biases near threshold. In the above threshold analysis, we use the simulation to illustrate 
how important parasitic effects are in affecting the modulation response and show that carrying out these experiments with optical modulation removes some but not all of the parasitics. The latter is the configuration used in this work to obtain the modulation response of the InGaAsN RWG lasers. With the help of the model we extract the resonance frequency and the damping and obtain relevant parameters such as the effective differential gain parameters. It is found that the RWG single quantum well InGaAsN lasers reach modulation bandwidths $f_{-3 \mathrm{~dB}} \approx 6 \mathrm{GHz}$ at $2.5 \mathrm{~mW}$ emitted power. These are the highest $f_{-3 \mathrm{~dB}}$ per facet output power levels reported for uncoated single quantum well RWG devices. Analysis of the modulation responses reveal that the resonance frequency and damping and not the transport pole control the bandwidth which is reduced with increased nitrogen content in the well.

This paper is structured as follows: Section II describes in detail the model used to analyze the frequency responses of the dilute nitride lasers. The model is based on rate equations and incorporates parasitics arising from the device structure and circuitry, which on the BA and RWG lasers investigated are very important. Section III provides details of the devices, their geometry and growth characteristics, and of the experimental tools used to obtain the below and above threshold frequency response of the dilute nitride lasers. Section IV presents the experimental results and discusses their interpretation via the use of the model described in Section II.

\section{ANALYSIS OF THE FREQUENCY RESPONSE}

In this section we describe a comprehensive model that simulates the small signal modulation response (FR) of InGaAsN $(N=0 ; 0.005)$ laser diodes intended to separate intrinsic from parasitic effects. The results of the model are used to fit the frequency response behavior measured experimentally below and above threshold. Analysis of the below threshold frequency response is used to extract the differential carrier lifetime and from it identify the carrier recombination mechanisms [2], [3]. Above threshold, the FR provides the laser resonance frequency, damping, and carrier transport parameters [7], [10], [18], [29]-[31] that determine the ultimate device bandwidth.

In most cases, FR measurements have been conducted on devices whose electrical parasitics have been minimized through careful design of the laser structure or the experiment, as in the case of optical modulation [4]-[6], [9]. For quantum well lasers the FR is simulated in this case by a 3-rate equation model involving the carrier populations in the well, barrier/separate confinement and photon populations. This model has been successfully used by Pikal et al. [3], to obtain the intrinsic well differential lifetime and from it analyze the mechanisms that affect $T_{0}$ on 1.3- $\mu \mathrm{m}$ InAsP buried heterostructure lasers, and by others to investigate limiting mechanisms in the above threshold frequency response [5], [9], [18], [32].

The 3-rate equation model is however, not sufficient to analyze the FR of the InGaAsN $(N=0 ; 0.005)$ RWG and BA lasers investigated in this work, due to the significant contribution of electrical parasitics. The parasitics add additional poles

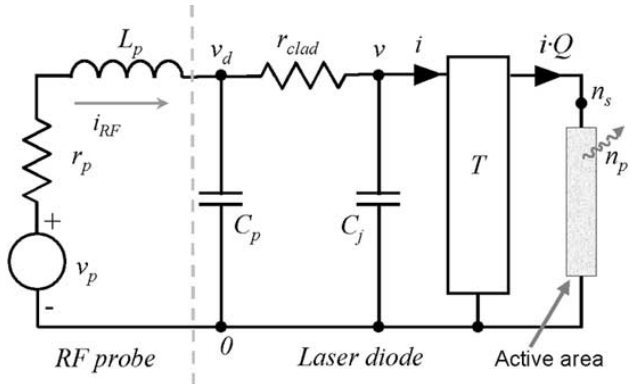

Fig. 1. Small signal circuit of the laser diode and electrical parasitics with its driving circuitry.

and zeroes that mask the processes that occur within the laser diode active region.

The most complete rate equation system that best describes our InGaAsN devices is represented by the schematic circuit of Fig. 1. At the left, the RF probe is given by its Thévenin open circuit voltage $v_{p}$ and complex inductive impedance $r_{p}+j 2 \pi f L_{p} . L_{p}$ and $r_{p}$ are the total inductance and resistance seen by the test laser device towards the RF probe. The laser diode has a parasitic capacitance $C_{p}$ between its contacts and a cladding series resistance $r_{\text {clad }}$. The capacitance $C_{j}$ represents the diode junction whose dependence with the diode bias voltage is described in Section II-A.

The box labeled "active area" represents the 3-rate equation model involving the small signal perturbations $n_{s}, n_{w}$ and $n_{p}$ to the barrier/separate confinement $(\mathrm{SCH})$, quantum well (QW) and photon reservoirs, respectively, characterized by corresponding steady-state carrier densities $N_{s}, N_{w}$, and $N_{p}$ [33].

The circuitry shown in Fig. 1 is described by a set of rate equations given by (1)-(5), valid for above and below threshold operation. The first two equations account for the electrical circuitry and parasitics by introducing the variables $v_{d}$ and $i_{\mathrm{RF}}$. Equations (3)-(5) describe the intrinsic processes in the laser active region in terms of $n_{s}, n_{w}$, and $n_{p}$

$$
\begin{aligned}
L_{p} \dot{i}_{\mathrm{RF}}= & v_{p}-r_{p} i_{\mathrm{RF}}-v_{d} \\
C_{P} \dot{v}_{d}= & i_{\mathrm{RF}}-\frac{v_{d}}{r_{\mathrm{clad}}}+\frac{v}{r_{\mathrm{clad}}} \\
\dot{n}_{s}= & Q\left(\frac{v_{d}}{r_{\mathrm{clad}}}-\frac{v}{r_{\mathrm{clad}}}-C_{j} \dot{v}\right) \\
& -\frac{n_{s}}{\tau_{\mathrm{cap}}}-\frac{n_{s}}{\tau_{s}}+\Gamma_{q} \frac{n_{w}}{\tau_{\mathrm{esc}}} \\
\dot{n}_{w}= & \frac{n_{s}}{\Gamma_{q} \tau_{\mathrm{cap}}}-\frac{n_{w}}{\tau_{w}}-\frac{n_{w}}{\tau_{\mathrm{esc}}} \\
& -\left[P \cdot n_{w}+\left(A_{p} \cdot P+\frac{1}{\Gamma \cdot \tau_{p}}\right) n_{p}\right] \\
\dot{n}_{p}= & \left(\beta \frac{n_{w}}{\tau_{w}}-\frac{n_{p}}{\tau_{p}}\right)+\left[\Gamma \cdot P\left(n_{w}+A_{p} \cdot n_{p}\right)\right] .
\end{aligned}
$$

In (3)-(5), $\tau_{s}, \tau_{w}$, and $\tau_{p}$ are the differential lifetime of the carriers in the SCH and well, and the photon lifetime, respectively. The current into the laser active region $i$ in Fig. 1 is given in (3) by the parenthesis term. The factor $Q$ is used to simplify 
notation as $Q=\eta_{\text {inj }} / q V_{s}$ where $V_{s}$ is the volume of the $\mathrm{SCH}$, and $\eta_{\text {inj }}$ is the current injection efficiency which is assumed constant and equal to its threshold value [34]. $\Gamma_{q}, \Gamma$, and $\beta$ are the ratio of the well and $\mathrm{SCH}$ volumes, the optical mode confinement factor and the spontaneous emission factor, whose values for the studied laser structures are found to be $0.02,0.018$ [14] and $\sim 8.7 \cdot 10^{-5}[33]$, respectively.

The bracketed terms in (4) and (5) that include $A_{p}$ and $P$, are described in Section II-B for above threshold biases as these vanish below threshold. On the contrary, the term between parentheses in (5) is zero above threshold, as described in the next subsection. The time constants $\tau_{\text {cap }}$ and $\tau_{\text {esc }}$ represent the processes of carrier capture into and escape out of the well. These time constants have not been measured for InGaAsN, but can be estimated. $\tau_{\text {esc }}$ at threshold, calculated from the thermionic electron/hole escape in the InGaAs and InGaAsN was found to be 50 ps and 5 ps, respectively [34]. As it will be shown in Section IV, an upper bound for $\tau_{\text {cap }}$ obtained from the measurements is found to be 8 ps for both InGaAs and InGaAsN lasers. We introduce the parameter $R$ to represent the well effective carrier capture given by $R=\tau_{\text {cap }} / \tau_{\text {esc }}$. $R$ plays a fundamental role in the interpretation of the carrier lifetime [3] and in the above threshold frequency response.

Equation (3) couples the electrical circuitry with the carrier densities by including the small signal voltage $v(t)$. We relate $v(t)$ and $n_{s}(t)$ by a frequency independent transmission network, with parameter $T=n_{s} / v$, similar to a transformer of ratio $T: 1 . T$ is such that the ratio $v / i$ calculated at $f \rightarrow 0$ (DC) from the steady-state solution of (3), (5) equals the $p$ - $n$ junction differential resistance $r_{d}$ at all bias currents. $T$ is fundamental in defining the coupling between the intrinsic active area dynamics and parasitics and external circuitry. Its values are estimated to be much larger above threshold than below threshold, which indicates a weaker impact of parasitics for the former, as it will be shown in Section II-B. A similar concept for the coupling introduced here was previously utilized to study the electrical impedance of InGaAs-GaAs QW lasers [32].

The frequency domain representation of (1)-(5) is written in (6) after replacing $v$ by $n_{s} / T$. For subthreshold operation, the elements $[4,5]$ and $[5,5]$ in (6), shown at the bottom of the page, are replaced by zero and $j \omega+1 / \tau_{p}$, respectively.
TABLE I

InGaAs AND InGaAsN LASERS PARAMETERS

\begin{tabular}{lcc}
\hline \hline \multicolumn{1}{c}{ Structure $\rightarrow$} & InGaAs & InGaAsN \\
\hline$\tau_{\text {esc,th }}$ & $50 \mathrm{ps}$ & $5 \mathrm{ps}$ \\
$\tau_{\text {cap }, \mathrm{hh}}$ & $<8 \mathrm{ps}$ & $<8 \mathrm{ps}$ \\
$\eta_{\text {inj }}$ & 0.8 & 0.7 \\
$\mathrm{I}_{\mathrm{th}}(\mathrm{RWG})$ & $6 \mathrm{~mA}$ & $13 \mathrm{~mA}$ \\
$\mathrm{I}_{\mathrm{th}}(\mathrm{BA})$ & $96 \mathrm{~mA}$ & $256 \mathrm{~mA}$ \\
$\mathrm{dP}_{0} / \mathrm{dI}(\mathrm{RWG})$ & $\sim 0.2 \mathrm{~W} / \mathrm{A}$ & $\sim 0.2 \mathrm{~W} / \mathrm{A}$ \\
$\tau_{\mathrm{s}}$ & $\sim 5 \mathrm{~ns}$ & $\sim 5 \mathrm{~ns}$ \\
$\Gamma_{\mathrm{q}}$ & 0.02 & 0.02 \\
$\Gamma$ & 0.018 & 0.018 \\
$\mathrm{QW}$ & $1 \times 60 \AA$ & $1 \times 60 \AA$ \\
\multicolumn{2}{c}{$\mathrm{C}_{\mathrm{p}}=20 \mathrm{pF}, \mathrm{C}_{\mathrm{j}}=5 \mathrm{pF}\left(\mathrm{RWG}, \mathrm{I}>\mathrm{I}_{\mathrm{th}}\right)$} \\
$\mathrm{r}_{\mathrm{s}}=5 \Omega, \mathrm{r}_{\mathrm{d}}=1-50 \Omega, \mathrm{L}_{\mathrm{s}}=1 \mathrm{nH}$ \\
\hline \multicolumn{2}{c}{}
\end{tabular}

Analytical solutions of the set of equations given in (6) for biases below and above threshold were obtained. These solutions, tailored to the specific case of InGaAsN lasers, are described in the following sections.

\section{A. Subthreshold Frequency Response}

The exact solution of (6) for below threshold biases $\left(\mathrm{I}_{\mathrm{th}}\right)$, i.e., obtained for $P=0$, yields a five-pole response with only one dominant pole $\tau$, as shown in

$$
\begin{aligned}
H_{\mathrm{sp}}(f) & \\
= & \frac{d P_{\mathrm{sp}} /\left.d v_{p}\right|_{f \rightarrow 0}}{(1+j 2 \pi f \tau)} \\
& \cdot \frac{1}{\left(1+j 2 \pi f \tau_{1}\right)\left(1+j 2 \pi f \tau_{2}\right)\left(1+j 2 \pi f \tau_{3}\right)\left(1+j 2 \pi f \tau_{p}\right)}
\end{aligned}
$$

In (7) $d P_{\mathrm{sp}} /\left.d v_{p}\right|_{f \rightarrow 0}$ represents the incremental ratio of laser spontaneous emission to the modulation voltage. The poles $\tau_{1}$ and $\tau_{2}$ are mainly influenced by parasitics and external circuitry while $\tau_{3}$ also depends on the laser intrinsic parameters. The numerical solution of (6) for InGaAsN lasers with parameters summarized in Table I, yields the reciprocal of $\tau_{1}, \tau_{2}, \tau_{3}$, and $\tau_{p}>2 \mathrm{GHz},>3 \mathrm{GHz},>3 \mathrm{GHz}$, and $\sim 50 \mathrm{GHz}$ in a bias range within a decade below $\mathrm{I}_{\mathrm{th}}$. At such high frequency these poles

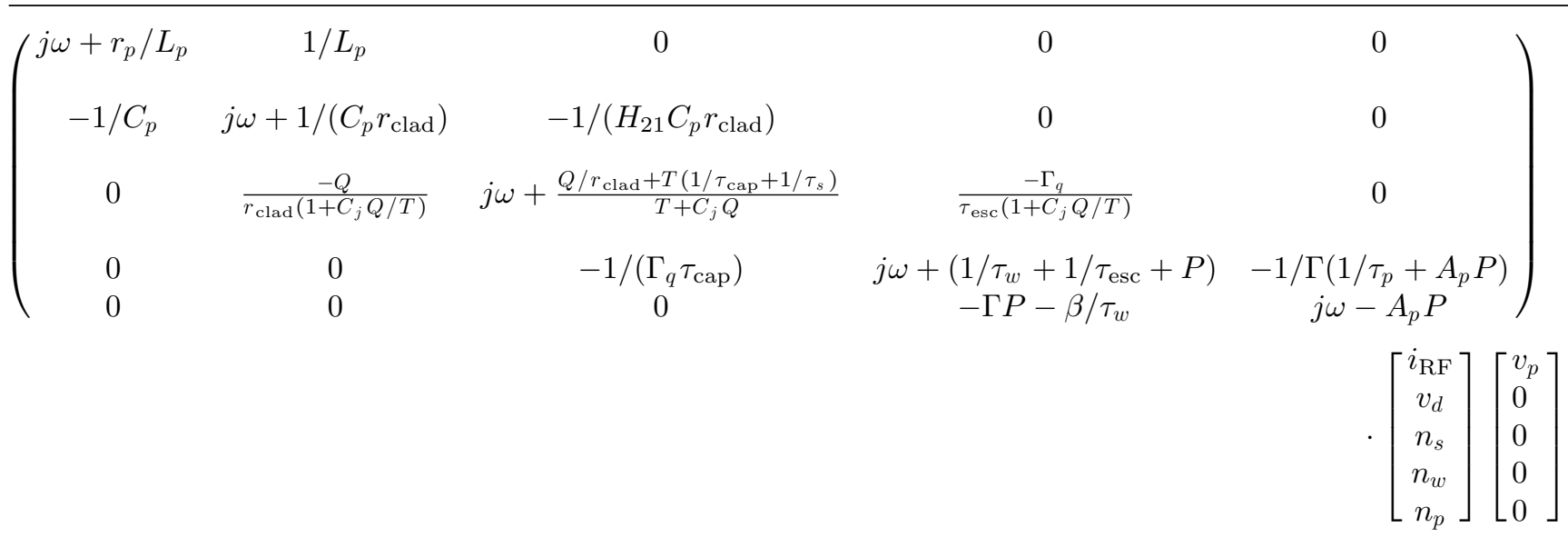




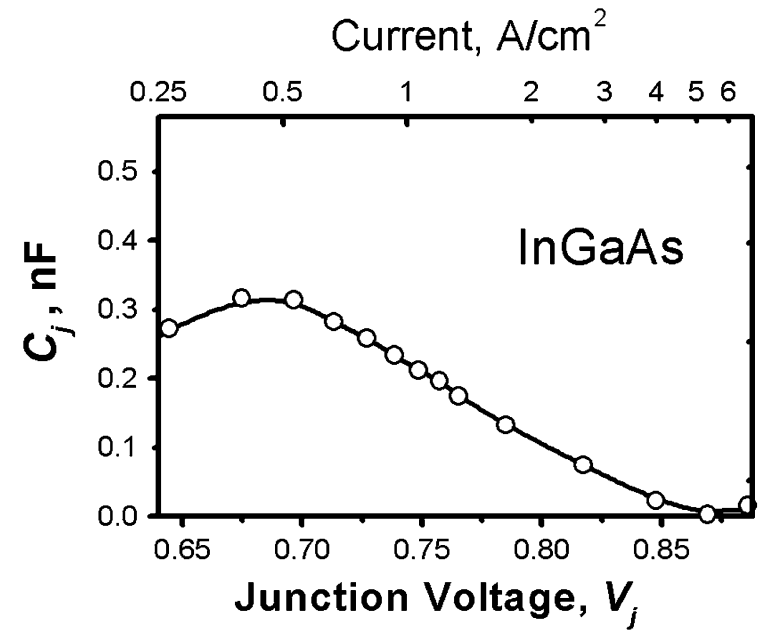

Fig. 2. Voltage dependent diode junction capacitance $C_{j}$, for a typical BA InGaAs laser diode.

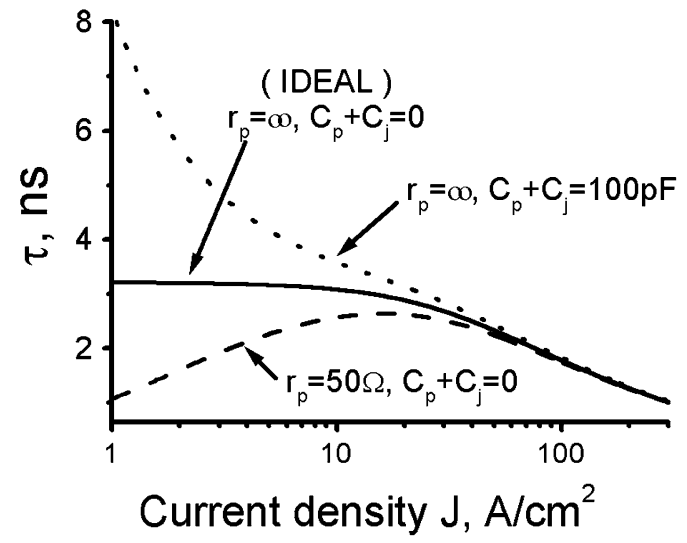

Fig. 3. Model simulation of the below threshold dominant pole $\tau$ versus $\mathbf{J}$ for three different cases. Ideal case-without parasitics $\left(\tau=\tau_{w}, C_{p}+C_{j}=\right.$ $\left.0, r_{p} \gg r_{d}\right)$. For $C_{p}+C_{j}=0, r_{p}=50 \Omega$. For $C_{p}+C_{j}=100 \mathrm{pF}, r_{p} \geq r_{d}$. (Other values are from Table I). $\tau_{w}$ is calculated from equation (13), using values of $\mathrm{A}=3 \cdot 10^{8} \mathrm{~s}^{-1}, \mathrm{~B}=10^{-13} \mathrm{~cm}^{-3} \mathrm{~s}^{-1}$, and $\mathrm{C}=9 \cdot 10^{-29} \mathrm{~cm}^{-6} \mathrm{~s}^{-1}$.

pose negligible effects in the $1 \mathrm{GHz}$ range of the measurements detailed in Section III-B. Similar values are found in InGaAs lasers.

The dominant pole $\tau$ extracted from the analytical solution of (6) is the one that mainly contains the information on the well differential lifetime $\tau_{w}$. We find that in the limit $r_{p} / r_{d} \rightarrow \infty, \tau$ can be expressed as $\tau \approx \tau_{w}+r_{d}\left(C_{p}+C_{j}\right)$. In most cases, as it occurs in the InGaAsN lasers, $C_{p}<C_{j}$ and is not bias dependent. Instead, $C_{j}$, behaves with bias similarly to the models developed by [35], [36]. Fig. 2 shows the behavior of $C_{j}$ versus bias voltage in the BA InGaAs laser diodes, extracted from device impedance measurements near the built-in junction voltage [37]. $C_{j}$ reaches its maximum at $V_{j}=0.7 \mathrm{~V}$, decaying at both sides and becoming constant as the lasers reach threshold. Similar traces of $C_{j}$ versus $V_{j}$ are found in the BA InGaAsN laser diodes.

It is instructive to analyze the behavior of $\tau$ for different cases of $r_{p}$ and $C_{p}+C_{j}$. This is shown in Fig. 3. When $r_{p}=50 \Omega$ and $C_{p}+C_{j}=0$, the solution of (6) predicts a reduction in $\tau$ with bias, as observed by Olshansky et al. [1]. When compensating the bias dependent diode impedance by setting $r_{p}>>r_{d}$ and having also $C_{p}+C_{j}=0$, Fig. 3 shows that $\tau$ saturates at low biases. In contrast with these results, $\tau$ continues to increase as the bias decreases if $C_{p}+C_{j} \neq 0$. This is the situation encountered in the lifetime of InGaAs and InGaAsN lasers discussed in Section IV.

The expression for $T$ obtained from the steady state solution $n_{s} / i$ of (3) through (5) and by knowing that $T^{-1} \cdot n_{s} / i=r_{d}$ is shown in

$$
T=\frac{Q}{r_{d}}\left(\frac{1}{\tau_{s}}+\frac{R}{\tau_{w}+\tau_{\mathrm{esc}}}\right)^{-1} .
$$

For the parameters given in Table I, $T$ is estimated to be $\sim 10^{18}-10^{19} \mathrm{~cm}^{-3} \cdot \mathrm{V}^{-1}$ for BA InGaAs and InGaAsN lasers at $0.1 \cdot J_{\mathrm{th}}-J_{\mathrm{th}}$. We assume in this case that $R$ is fixed at its threshold value of $8 / 50$ and $8 / 5$ for InGaAs and InGaAsN respectively.

\section{B. Frequency Response Above Threshold}

The model in (6) is solved by considering $P$, given in (9), to be a function of the emitted power per facet $P_{0}$, mirror losses $\alpha_{m}$, photon energy in vacuum $h \nu$ and the gain parameter $a=$ $d g / d N_{w}$ [33]. $A_{p}$ is negative and proportional to the ratio of $a_{p}$ and $a$, where $a_{p}=d g / d N_{p}$, as shown in

$$
\begin{aligned}
P & =\frac{2 a P_{0} \Gamma}{\alpha_{m} h v V_{s}} \\
A_{p} & =\Gamma a_{p} / a .
\end{aligned}
$$

The expression of the transfer function $H_{\mathrm{st}}(f)$ above threshold contains five poles

$$
\begin{aligned}
H_{\mathrm{st}}(f)=\frac{1}{\left(1+j 2 \pi f \tau_{1}\right)\left(1+j 2 \pi f \tau_{2}\right)} \\
\cdot \frac{d P_{0} /\left.d v_{p}\right|_{f \rightarrow 0}}{\left(1+j 2 \pi f \tau_{c}\right)\left(f_{r}^{2}-f^{2}+j f \gamma\right)} .
\end{aligned}
$$

The two principal poles, $f=\alpha+j \beta$ and $f=\alpha-j \beta$, arise from the stimulated process in the laser active area and are customarily expressed by the relaxation frequency $f_{r}=\sqrt{\alpha^{2}+\beta^{2}}$ and damping $\gamma=2 \alpha$. In the parasitic-free case, the third pole $1 / 2 \pi \tau_{c}$ represents the combined effects of the well quantum capture and carrier diffusion along the SCH [38], however this is not the case when parasitics are important. In fact, our model shows that $f_{r}, \gamma$, and $\tau_{c}$ depend on $L_{p}, C_{p}, C_{j}, r_{s}$, and $r_{p}$. The two other poles $1 / 2 \pi \tau_{1}$ and $1 / 2 \pi \tau_{2}$ arise mainly from the external circuit and parasitics. Because they typically fall in the 1-10 GHz range, these poles can be erroneously interpreted as $1 / 2 \pi \tau_{c}$. In the typical above threshold FR of InGaAsN lasers we find that the effects of $f_{r}, \gamma, \tau_{1}, \tau_{2}$, and $\tau_{c}$ are mixed together in the range of frequencies below $10 \mathrm{GHz}$. Electrical modulation traces predicted by the model with the laser parameters of Table I are shown in Fig. 4.

The analytical expression of $T$ above threshold is obtained from (3) and (5) as described for the below threshold case. However, its expression above threshold is more complex. $T$ depends on $P_{0}, a, R, a_{p}, r_{d}$, and $\eta_{\mathrm{inj}}$. By assuming fixed values 


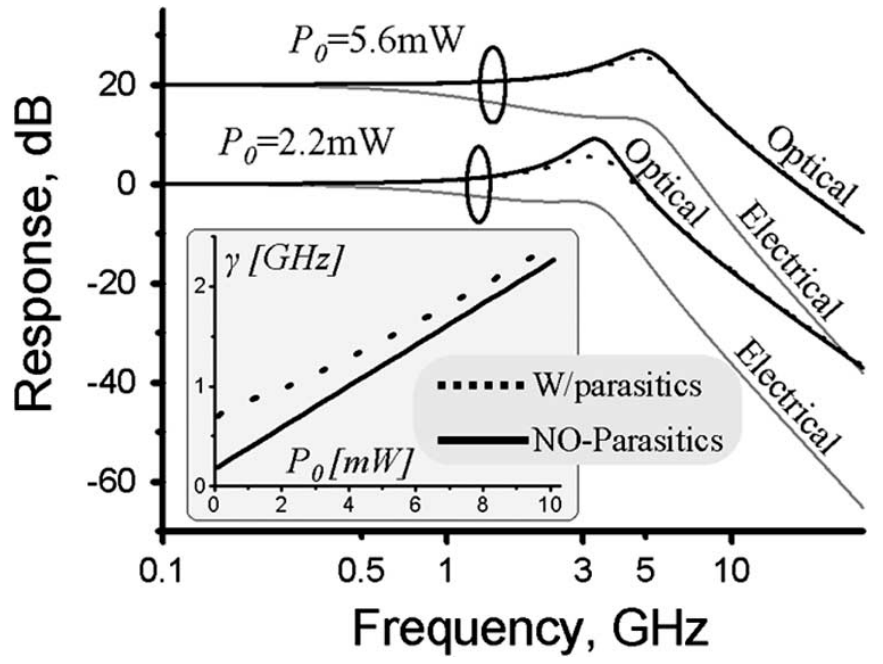

Fig. 4. Calculated frequency responses of InGaAs above threshold for optical (black solid line) and electrical (gray solid line) modulation at $P_{0}=2.2 \mathrm{~mW}$ and $P_{0}=5.6 \mathrm{~mW}$. The parasitics significantly affect the electrical modulation responses while only increase the damping in the optical modulation responses (black dotted line). Inset: $\gamma-P_{0}$ with and without the contributions of parasitics.

of $\tau_{\text {cap }}$ and $\tau_{\text {esc }}$ from Table I and, adopting $A_{p} \sim-0.8$ for InGaAs QW lasers as in [33], we find typical values of $T$ in the order of $\sim 10^{19}-10^{20} \mathrm{~cm}^{-3} V^{-1}$ in the range $P_{0}=1-7 \mathrm{~mW}$ for the InGaAs and InGaAsN RWG-lasers. The larger value of $T$ compared with that below threshold indicates a weaker coupling of the parasitics and the laser active region above threshold.

\section{Optical Modulation}

We consider here the case of above frequency response measurements obtained by modulating the carriers in the laser active region via the use of a tunable optical source. In practice, optical modulation is a way to minimize the effects of electrical parasitics [18]. Within the model, this is equivalent to adding an optical perturbation $S_{o}$ in (6) and turning off $v_{p}$. The frequency response obtained from the model (6) is shown in (12), where the factor $H_{z p}$ includes two new zeroes $\tau_{z 1}$ and $\tau_{z 2}$ and the two poles $\tau_{1}$ and $\tau_{2}$ from (11). Interestingly, it is found that for the typical parameters of InGaAs and InGaAsN lasers, $\tau_{z 1}$ and $\tau_{z 2}$ nearly cancel out with $\tau_{1}$ and $\tau_{2}$, thereby yielding $H_{z p} \rightarrow 1$ and a response that only depends on $f_{r}, \gamma$, and $\tau_{c}$

$$
H_{\mathrm{opt}}(f)=H_{z p} \cdot \frac{d P_{0} /\left.d S_{o}\right|_{f \rightarrow 0}}{\left(1+j 2 \pi f \tau_{c}\right)\left(f_{r}^{2}-f^{2}+j f \gamma\right)} .
$$

The simulated optical frequency responses obtained using the full model with and without parasitics are plotted in Fig. 4 and are also compared with FR calculated from electrical modulation. Notice that the parasitics affect $\gamma$ but not $f_{r}$. The former increasing with parasitics as is plotted in the inset of Fig. 4. This contrast with the electrical modulation frequency responses where $\gamma$ and $f_{r}$ are masked by the additional poles arising from the parasitics, as predicted by (11). These results conclusively show that parasitics must be taken into account when performing the fit of FR to obtain meaningful gain compression factor $\varepsilon$ and differential gain parameters $a, a_{p}$ [33].
Based on the findings of the model, we focus the analysis on the behavior of $f_{r}$ versus $P_{0}$ obtained from the optical FR of InGaAsN, as it directly reflects the laser intrinsic dynamics, as discussed in Section IV-B.

\section{DESCRIPTION OF EXPERIMENTS}

\section{A. Laser Diode Characteristics}

The FR measurements were carried out in identical laser diodes that only differ in the presence of nitrogen in the QW. The devices, were grown by MOCVD and feature a $\mathrm{In}_{0.4} \mathrm{Ga}_{0.6} \mathrm{As}$ or $\mathrm{In}_{0.4} \mathrm{Ga}_{0.6} \mathrm{As}_{0.995} \mathrm{~N}_{0.005}$ single quantum well of $L_{z}=60 \AA$ which is surrounded by two tensile strain $\mathrm{GaAs}_{0.85} \mathrm{P}_{0.15}$ barriers with $100 \AA$ GaAs spacers in between. This structure is embedded into a $3000 \AA$ GaAs separate confinement region ( $\mathrm{SCH}$ ) surrounded by the $\mathrm{p}^{+}$and $\mathrm{n}^{+}$AlGaAs claddings which form the laser diode [14]. The respective peak emissions of the $\mathrm{In}_{0.4} \mathrm{Ga}_{0.6}$ As and $\mathrm{In}_{0.4} \mathrm{Ga}_{0.6}$ As ${ }_{0.995} \mathrm{~N}_{0.005}$ single quantum wells are 1.21 and $1.29 \mu \mathrm{m}$ at $20{ }^{\circ} \mathrm{C}$.

The InGaAs and InGaAsN devices used for frequency response above threshold are RWG devices, with a $2.5-\mu \mathrm{m}$ strip width ${ }^{1}$ and 500- $\mu$ m length. Similar BA InGaAs (InGaAsN) lasers with $100-\mu \mathrm{m}$-width stripe by $750-\mu \mathrm{m}$ (1 $\mathrm{mm})$ length and $J_{\mathrm{th}}=125 \mathrm{~A} / \mathrm{cm}^{2}\left(256 \mathrm{~A} / \mathrm{cm}^{2}\right)$ were used to obtain the frequency response below threshold. Typical threshold currents are 6 and $96 \mathrm{~mA}$ for the InGaAs RWG and BA structures, and 13 and $256 \mathrm{~mA}$ for the dilute nitride counterparts, respectively.

\section{B. Below Threshold FR Measurements}

In these experiments the BA lasers are driven by an RF probe of very high output impedance $r_{p}>>r_{d}$ [39] that is connected to the output port of an Agilent 8753 network analyzer (VNA). We identified two measurement regimes depending upon the current, and modified the setup accordingly. At low currents, where Joule heating is not significant, the VNA combined the frequency swept sine wave with the DC bias current. The laser output was measured by an avalanche photodiode (APD) whose output signal was connected to an amplifier and then returned to the VNA input port. In the high current regime, for bias currents exceeding $30 \mathrm{~A} / \mathrm{cm}^{2}$, the frequency response was measured under pulse conditions in order to reduce the active area Joule heating, thus assuring that all measurements were performed at a constant active area temperature $\left(\mathrm{T}=20^{\circ} \mathrm{C}\right)$. The frequency of the sine wave for both setups was swept from $0.1 \mathrm{MHz}$ to up to $1 \mathrm{GHz}$, at an amplitude level that produced a modulation index of $5 \%$ or less in the laser current.

\section{Above Threshold FR Measurements}

In these experiments, we used both electrical and optical modulation. The electrical modulation technique, the same as discussed above, yielded FR traces that were very strongly affected by additional poles, as predicted by the model of Section II-B.

\footnotetext{
${ }^{1}$ The stripe width was obtained from the near field pattern of the RWG lasers above threshold.
} 

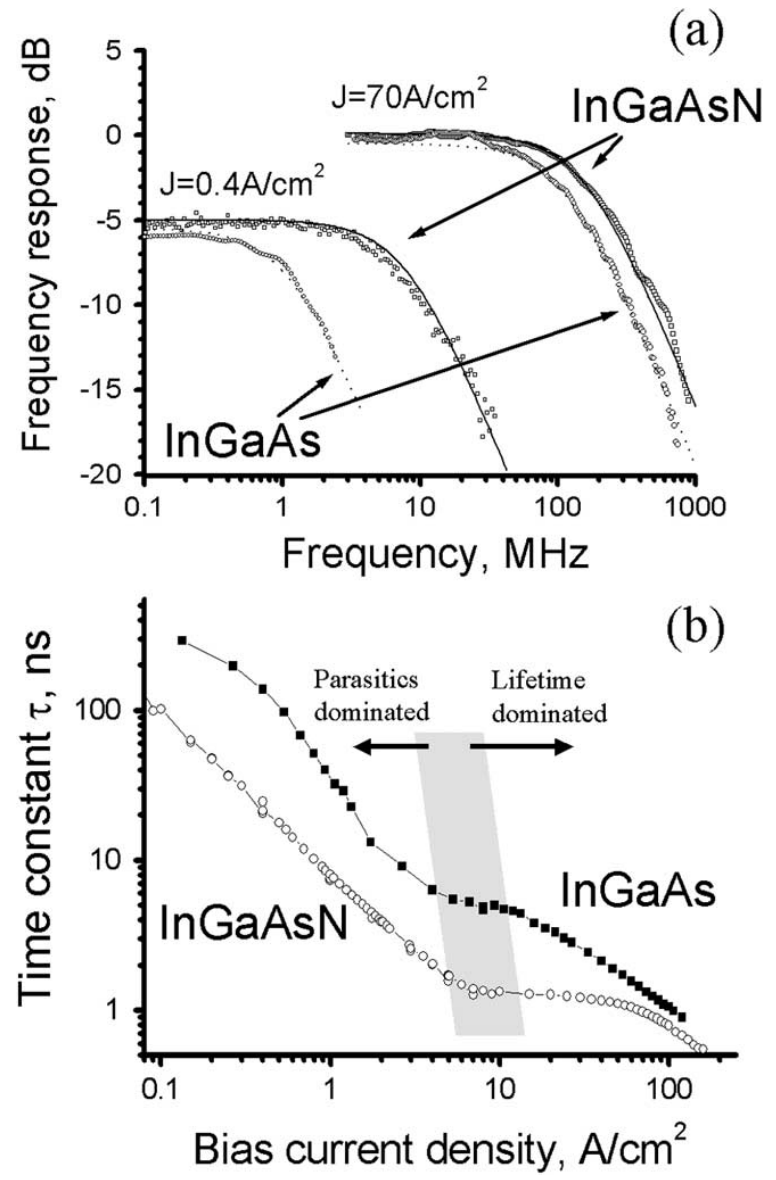

Fig. 5. (a) Below threshold frequency responses of InGaAs and InGaAsN laser diodes at $J=0.4 \mathrm{~A} / \mathrm{cm}^{2}$ and $J=70 \mathrm{~A} / \mathrm{cm}^{2}$. The solid lines are a fit with (7). (b) Variation of the dominant pole $\tau$ with bias up to threshold. The shaded area separates the regions where the lifetime is dominated by parasitics (left) and by the carrier recombination lifetime (right).

For the optical modulation we used a method developed in our lab [18] whereby a small signal perturbation is produced by short optical pulses impinging through one of the facets into the laser active area. The laser response was collected by a detector and measured with a spectrum analyzer, as previously described [18]. A Ti-Sapphire laser $(\lambda=795 \mathrm{~nm})$ was utilized as the optical excitation source. After being stretched to $0.5 \mathrm{ps}$ and attenuated to $20 \mathrm{~mW}$, the Ti-Sapphire pulses were focused on the back facet of the laser diode by a lens attached to an $X-Y-Z$ positioner. Small signal conditions were ensured by reducing the intensity of the optical signal to a level where the laser diode output and the peak wavelength shifted less than 5\% and $1 \AA$, respectively.

The RWG test lasers were dc biased. They were mounted on a baseplate whose temperature was adjusted to maintain constant the active area temperature, which was set to $21^{\circ} \mathrm{C}$. The condition of constant active area temperature was achieved by reducing the baseplate temperature, at a rate of $3.06^{\circ} \mathrm{C} / \mathrm{mA}$, to ensure the emission wavelength remained at $1.29 \mu \mathrm{m}$. The frequency span of the measurements was determined by setting the spectrum analyzer to a $70-\mathrm{MHz}-10-\mathrm{GHz}$ sweep range, with an intermediate frequency of $30 \mathrm{kHz}$.
TABLE II

RECOMBINATION COEFFICIENTS FOR InGaAs AND InGaAsN RETRIEVED FROM THE FIT OF $\tau_{w}(J)$

\begin{tabular}{lccc}
\hline \hline Structure & $\mathrm{A}\left[\mathrm{s}^{-1}\right]$ & $\mathrm{B}\left[\mathrm{cm}^{3} \mathrm{~s}^{-1}\right]$ & $\mathrm{C}\left[\mathrm{cm}^{6} \mathrm{~s}^{-1}\right]$ \\
\hline InGaAs & $2.1 \cdot 10^{8}$ & undetermined & $7.7 \cdot 10^{-29}$ \\
InGaAsN & $7.7 \cdot 10^{8}$ & undetermined & $3.1 \cdot 10^{-28}$ \\
\hline \hline
\end{tabular}

\section{RESULTS AND DISCUSSION}

\section{A. Subthreshold Frequency Responses}

Fig. 5(a) shows the frequency response traces obtained in InGaAs and InGaAsN laser structures at bias current densities of $0.4 \mathrm{~A} / \mathrm{cm}^{2}$ and $70 \mathrm{~A} / \mathrm{cm}^{2}$. These traces are representative of the behavior observed at all biases. The single dominant pole $1 / 2 \pi \tau$ was extracted by minimum squares fit of the experimental traces with (7).

Fig. 5(b) plots $\tau$ versus the bias current density $J$ for both lasers. Two different features are observed: 1) $\tau$ is smaller in the InGaAsN than in InGaAs lasers, and 2) after looking like it may saturate at around $10 \mathrm{~A} / \mathrm{cm}^{2}$, the lifetime then continues to increase as the bias is reduced further. The former observation is very important as it suggests that the carrier recombination rate is increased in the dilute nitride lasers, while the latter indicates that $\tau$ is affected by the device parasitics below $7-10 \mathrm{~A} / \mathrm{cm}^{2}$. Below this current range, $r_{d} \cdot C_{j}$ is much larger than $\tau_{w}$ and masks $\tau_{w}$ in the InGaAs and InGaAsN laser diodes. A similar behavior is evidenced from impedance measurements.

To obtain the dependence of $\tau_{w}$ on carrier density and extract from it the recombination parameters, $\mathrm{A}, \mathrm{B}$ and $\mathrm{C}$ we simultaneously fitted $\tau$ and current $J\left(N_{w}\right)$ using (13) and (14), respectively:

$$
\begin{aligned}
\frac{1}{\tau_{w}}= & \frac{d R\left(N_{w}\right)}{d N_{w}}=A+2 B N_{w}+3 C N_{w}^{2} \\
I= & 1 / Q\left[\Gamma_{q}\left(A N_{w}+B N_{w}^{2}+C N_{w}^{3}\right)\right. \\
& \left.+\left(A_{s} N_{s}+B_{s} N_{s}^{2}+C_{s} N_{s}^{3}\right)\right] .
\end{aligned}
$$

The values of the bias independent $\eta_{\text {inj }}$ and hence $Q$ and $R$ are given in Table I for InGaAs and InGaAsN. The fit allowed extraction of $A$ with an uncertainty of 5-10\%, and of $C$ with a $40 \%-60 \%$ error. The fit was not sensitive to $B$, mainly because after removing the region dominated by the parasitics there was only a decade left in current to extract $B$ from $\tau(J)$. The values of $A, B$, and $C$ retrieved from the fit are summarized in Table II. Notice the almost four time increase in $A$ with a $0.5 \%$ addition of nitrogen in the well.

A plot of $\tau$ versus $N_{w}$ in Fig. 6 distinctively shows the contribution of A, evidenced by the plateau of $\tau_{w}\left(N_{w}\right)$ in InGaAs for $N_{w}=(2-4) \times 10^{17} \mathrm{~cm}^{-3}$ and in InGaAsN for $N_{w}=(1-5) \cdot 10^{17} \mathrm{~cm}^{-3}$. Beyond these ranges and up to threshold the reduction of $\tau_{w}$ is due to the contribution of the term $1 / C N^{2}$.

The significant contribution of $A$ to the differential lifetime reflects on the diode's current density which is found to be dominated by the $A \cdot N_{w}$ term in (14) even at threshold in 


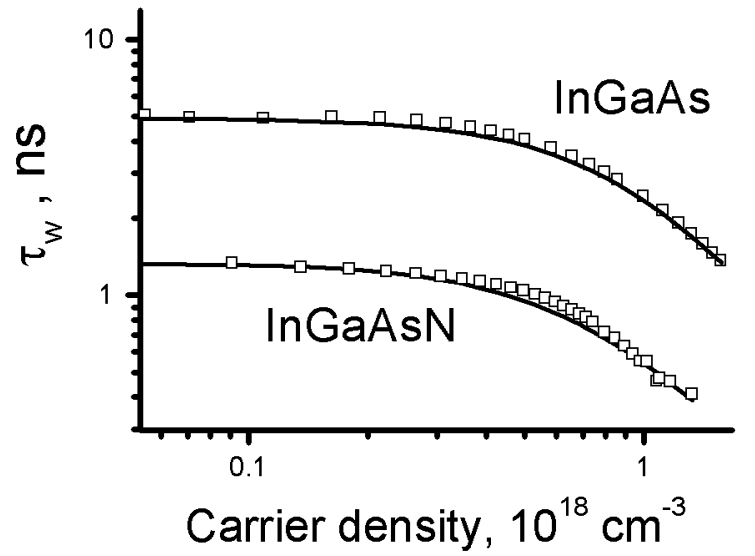

Fig. 6. $\tau_{w}$ versus $N_{w}$ for InGaAs and InGaAsN lasers extracted from the behavior $\tau-J$ of Fig. 5(b). The solid lines correspond to the fit with (13) and $A, B$, and $C$ given in Table II.

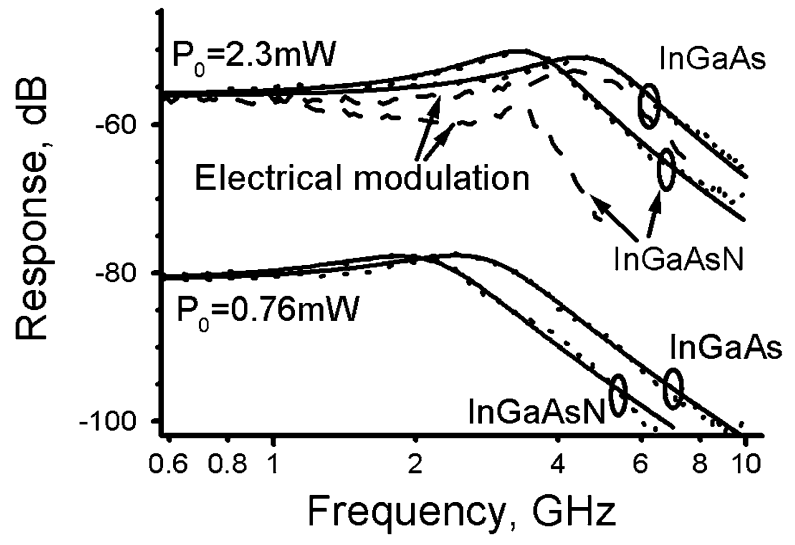

Fig. 7. Frequency responses of RWG InGaAs and InGaAsN lasers, for $P_{0}=$ $0.76 \mathrm{~mW}$ and $P_{0}=2.3 \mathrm{~mW}$, obtained with electrical (dotted lines) and optical (solid lines) modulation.

InGaAsN lasers [37]. Similarly, $A \cdot N_{w}$ also plays an important role in the recombination current of InGaAs. This may be a result of higher defect incorporation in the InGaAs active layer, which is grown at low temperature $\left(530^{\circ} \mathrm{C}\right)$. These results are in very good agreement with recent theoretical predictions that assess the impact of nitrogen on carrier recombination on InGaAsN lasers [25].

\section{B. Frequency Response Above Threshold}

The frequency responses above threshold measured on InGaAs and InGaAsN RWG lasers for optical modulation are shown in Fig. 7 for $P_{0}=0.76 \mathrm{~mW}$ and $P_{0}=2.3 \mathrm{~mW}$. The traces at $P_{0}=2.3 \mathrm{~mW}$ are compared with those obtained by electrical modulation, under the same experimental conditions. Notice in the electrical modulation traces the presence of an extra pole associated with a time constant of $170 \mathrm{ps}(30 \mathrm{ps})$ for the InGaAs (InGaAsN) lasers that has nothing to do with the transport pole $\tau_{c}$ in (12), as it does not appear in the optical modulation traces, but rather it corresponds to either $\tau_{1}$ or $\tau_{2}$ in (11). Interestingly, the absence of these poles in the optical modulation traces in a $10 \mathrm{GHz}$ bandwidth, as observed from Fig. 7, indicates that the influence of the transport pole $1 / 2 \pi \tau_{c}$

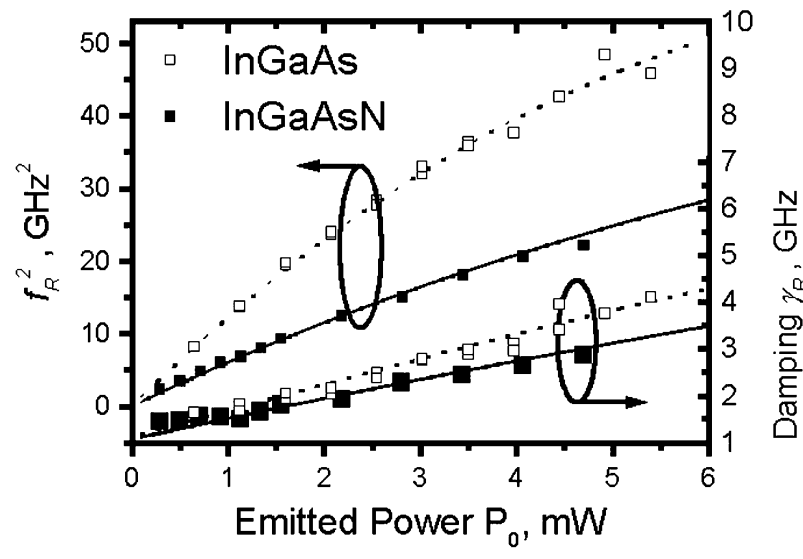

Fig. 8. Square of the resonance frequency, $f_{r}^{2}$, and damping $\gamma$, versus emitted power $P_{0}$ for RWG InGaAs and InGaAsN lasers.

is less than $1 \mathrm{~dB}$ in the $10 \mathrm{GHz}$ thus $\tau_{c}<8$ ps. We conclude that, $\tau_{c}$ does not impose a limit in the bandwidth of the RWG InGaAs and InGaAsN lasers.

The modulation traces of Fig. 7 also show that at optical powers corresponding to moderate biases above threshold, the single-well InGaAsN lasers reach $f_{3 \mathrm{~dB}} \approx 6 \mathrm{GHz}$ $\left(P_{0}=2.5 \mathrm{~mW}\right)$. These results suggest that by adding a second well, thereby enhancing $a=d g / d N_{w}$, the $1.3-\mu \mathrm{m} \mathrm{InGaAsN}$ RWG lasers can extend their bandwidth beyond the OC-48 telecom standard [21], [22], [24].

The modulation bandwidth of the InGaAsN is mainly controlled by $f_{r}$ and $\gamma$. Fig. 8 shows the $f_{r}^{2}$ and $\gamma$ versus $P_{0}$ behavior retrieved from the fit of optical modulation traces. There is a sublinear dependence of $f_{r}^{2}$ with $P_{0}\left(P_{0}=0-3 \mathrm{~mW}\right)$. Notice also the smaller $f_{r}^{2}-P_{0}$ slope in the InGaAsN with respect to InGaAs lasers. The reduction of $\sim 2$ in the $f_{r}^{2}-P_{0}$ slope near threshold in InGaAsN compared to InGaAs coincides with values obtained from recent relative intensity noise measurements (RIN) in similar structures [23]. Taking into account, as shown in Section II-B, that $f_{r}$ is not affected by the parasitics, we fitted $f_{r}^{2}$ versus $P_{0}$ using the solution of the model represented by (6) and leaving $a$ and $R$ as variables. The results of the fit, shown by the traces in Figs. 7 and 8, required the effective differential gain $a /(1+R)$ to vary as shown in Fig. 9. At threshold, the fit yielded values of $a /(1+R)=1.7$. $10^{-15} \mathrm{~cm}^{-2}$ and $0.85 \cdot 10^{-15} \mathrm{~cm}^{-2}$ in the RWG InGaAs and InGaAsN lasers respectively. The sublinear behavior of $f_{r}^{2}-P_{0}$ may arise from partial heating in the active area at the higher values of $P_{0}$, or due to quasi-Fermi levels depining [40] that impacts on both $R$ and $a$.

The analysis of the variation of $\gamma$ versus $P_{o}$ is not as direct as that of $f_{r}$ as $\gamma$ significantly depends on parasitics, as demonstrated in Section II-B and shown in the inset of Fig. 4. We conclude from the data of Fig. 8 that the insensitivity of $\gamma$ versus $P_{0}$ to the change in the nitrogen content is due to the contribution of parasitics. In spite of this, one can use the data of Fig. 8 to obtain an upper bound for $a_{p}$, from the slope of the $\gamma-P_{0}$ trace, considering that the corresponding parasitic-free $\gamma-P_{0}$ slope would be smaller as predicted by the model. In 


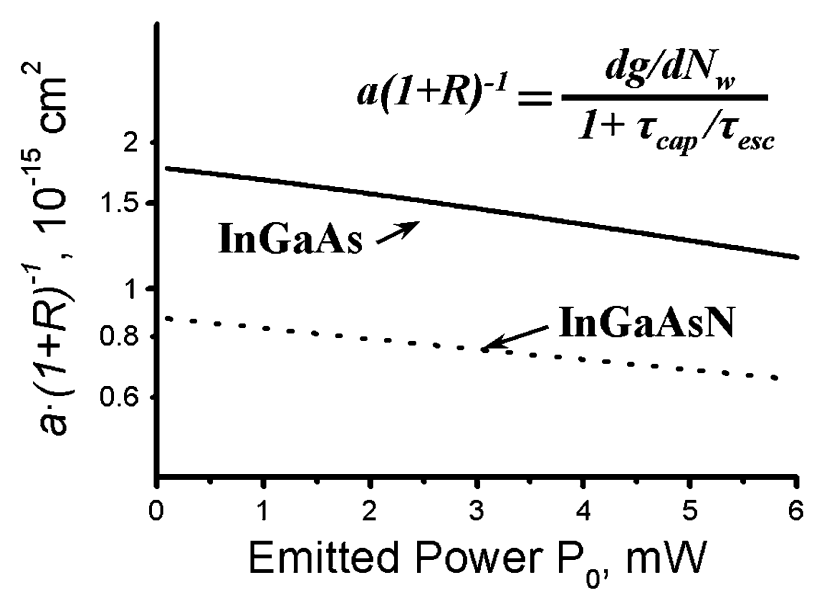

Fig. 9. Plot of the effective differential gain $d g / d N_{w} /\left(1+\tau_{\text {cap }} / \tau_{\text {esc }}\right)$ obtained from the fit of $f_{r}^{2}-P_{0}$ with the solution of the model for InGaAs and InGaAsN RWG lasers. The near threshold values of the effective differential gain are found as $1.7 \cdot 10^{-15} \mathrm{~cm}^{-2}$ and $0.85 \cdot 10^{-15} \mathrm{~cm}^{-2}$, respectively.

this case, and for the values of $a /(1+R)$ of Fig. 9, we find $\left|a_{p}\right|<10^{-13} \mathrm{~cm}^{2}$ in both laser structures.

\section{CONCLUSION}

We have developed a complete model of the frequency response of dilute nitride lasers based on a rate equation analysis that incorporates electrical and device parasitics. The solution of the model is used to analyze the below and above threshold frequency responses of InGaAsN lasers with different nitrogen content in the well. The model provides insight on the significant contribution of the parasitics to the carrier lifetime obtained from the below threshold responses at different biases, and to the frequency bandwidth of the lasers. The below threshold analysis revealed the increased contribution of monomolecular recombination in shaping the carrier recombination of InGaAsN lasers even at biases near threshold. From the above threshold analysis we have determined the single-well InGaAsN lasers reach $-3 \mathrm{~dB}$ bandwidths of $\sim 6 \mathrm{GHz}$ at moderate powers. Furthermore, we showed that the bandwidth is mainly controlled by the resonance frequency and damping, the latter being significantly influenced by parasitics. Using the model we explained the reduction in the bandwidth with nitrogen content in the well is likely to arise mainly from the combined effect of a reduction in the differential gain or an increase in the effective capture $\mathrm{R}=\tau_{\text {cap }} / \tau_{\text {esc }}$. The apparent insensitivity of the damping to nitrogen was ascertained to the more significant contribution of the parasitics.

\section{ACKNOWLEDGMENT}

L. J. Mawst acknowledges Dr. C. Pinzone from Ahura Corporation.

\section{REFERENCES}

[1] R. Olshansky, C. B. Su, J. Manning, and W. Powazinik, "Measurement of radiative and nonradiative recombination rates in InGaASp and AlGaAs light-sources," IEEE J. Quantum Electron., vol. QE-20, no. 8, pp. 838854, Aug. 1984.
[2] G. E. Shtengel, D. A. Ackerman, and P. A. Morton, "True carrier lifetime measurements of semiconductor-lasers," Electron. Lett., vol. 31, pp. 1747$1748,1995$.

[3] J. M. Pikal, C. S. Menoni, H. Temkin, P. Thiagarajan, and G. Y. Robinson, "Carrier lifetime and recombination in long-wavelength quantum-well lasers," IEEE J. Select. Topics Quantum Electron., vol. 5, no. 3, pp. 613619, May/Jun. 1999.

[4] J. Eom, C. B. Su, J. Lacourse, and R. B. Lauer, "Simultaneous measurement of spontaneous emission rate, nonlinear gain coefficient, and carrier lifetime in semiconductor-lasers using a parasitic-free optical modulation technique," Appl. Phys. Lett., vol. 56, pp. 518-520, 1990.

[5] D. Vassilovski, T. C. Wu, S. Kan, K. Y. Lau, and C. E. Zah, "Unambiguous determination of quantum capture, carrier diffusion, and intrinsic effects in quantum-well laser dynamics using wavelength-selective optical modulation," IEEE Photon. Technol. Lett., vol. 7, no. 7, pp. 706-708, Jul. 1995.

[6] R. Paiella, G. Hunziker, K. J. Vahala, and U. Koren, "Measurement of the interwell carrier transport lifetime in multiquantum-well optical amplifiers by polarization-resolved four-wave mixing," Appl. Phys. Lett., vol. 69, pp. 4142-4144, 1996.

[7] I. Esquivias, S. Weisser, B. Romero, J. D. Ralston, and J. Rosenzweig, "Carrier dynamics and microwave characteristics of GaAs-based quantum-well lasers," IEEE J. Quantum Electron., vol. 35, no. 4, pp. 635646, Apr. 1999

[8] M. Dutta and M. A. Stroscio, Advances in Semiconductor Lasers and Applications to Optoelectronics. Singapore: World Scientific, 2000.

[9] X. Jin and S. L. Chuang, "Microwave modulation of a quantum-well laser with and without external optical injection," IEEE Photon. Technol. Lett., vol. 13, no. 7, pp. 648-650, Jul. 2001.

[10] P. Bhattacharya and S. Ghosh, "Tunnel injection $\mathrm{In}_{0.4} \mathrm{Ga}_{0.6} \mathrm{As} / \mathrm{GaAs}$ quantum dot lasers with $15 \mathrm{GHz}$ modulation bandwidth at room temperature," Appl. Phys. Lett., vol. 80, pp. 3482-3484, 2002.

[11] B. Borchert, A. Y. Egorov, S. Illek, M. Komainda, and H. Riechert, "1.29 $\mu \mathrm{m}$ GalnNAs multiple quantum-well ridge-waveguide laser diodes with improved performance," Electron. Lett., vol. 35, pp. 2204-2206, 1999.

[12] K. D. Choquette, J. F. Klem, A. J. Fischer, O. Blum, A. A. Allerman, I. J. Fritz, S. R. Kurtz, W. G. Breiland, R. Sieg, K. M. Geib, J. W. Scott, and R. L. Naone, "Room temperature continuous wave InGaAsN quantum well vertical-cavity lasers emitting at $1.3 \mu \mathrm{m}$," Electron. Lett., vol. 36, pp. 1388-1390, 2000.

[13] A. W. Jackson, R. L. Naone, M. J. Dalberth, J. M. Smith, K. J. Malone, D. W. Kisker, J. F. Klem, K. D. Choquette, D. K. Serkland, and K. M. Geib, "OC-48 capable InGaAsN vertical cavity lasers," Electron. Lett., vol. 37, pp. 355-356, 2001.

[14] N. Tansu, N. J. Kirsch, and L. J. Mawst, "Low-threshold-current-density 1300-nm dilute-nitride quantum well lasers," Appl. Phys. Lett., vol. 81, pp. 2523-2525, 2002.

[15] D. Gollub, S. Moses, M. Kamp, and A. Forchel, "1.4 $\mu$ m continuouswave GaInNAs distributed feedback laser diodes," Electron. Lett., vol. 39, pp. 1815-1816, 2003.

[16] J. Y. Yeh, N. Tansu, and L. J. Mawst, "Long wavelength MOCVD grown InGaAsN-GaAsN quantum well lasers emitting at 1.378-1.41 $\mu \mathrm{m}$," Electron. Lett., vol. 40, pp. 739-741, 2004.

[17] S. R. Bank, M. A. Wistey, L. L. Goddard, H. B. Yuen, V. Lordi, and J. S. Harris, "Low-threshold continuous-wave 1.5- $\mu \mathrm{m}$ GaInNAsSb lasers grown on GaAs," IEEE J. Quantum Electron., vol. 40, no. 6, pp. 656-664, Jun. 2004.

[18] O. Anton, G. Vaschenko, D. Patel, G. Y. Robinson, C. S. Menoni, and J. Pikal, "Small signal response of $1.3 \mathrm{~mm}$ InAsP/InGaAsP quantum well laser diodes obtained with a THz-bandwidth frequency comb," IEEE J. Quantum Electron., vol. 40, no. 8, pp. 982-988, Aug. 2004.

[19] M. Kondow, K. Uomi, A. Niwa, T. Kitatani, S. Watahiki, and Y. Yazawa, "GaInNAs: A novel material for long-wavelength-range laser diodes with excellent high-temperature performance," Jpn. J. Appl. Phys., vol. 35, pp. 1273-1275, 1996.

[20] M. Kondow, T. Kitatani, S. Nakatsuka, M. C. Larson, K. Nakahara, Y. Yazawa, M. Okai, and K. Uomi, "GaInNAs: A novel material for long-wavelength semiconductor lasers," IEEE J. Sel. Topics Quantum Electron., vol. 3, no. 3, pp. 719-730, Jun. 1997.

[21] B. Borchert, A. Y. Egorov, S. Illek, and H. Riechert, "Static and dynamic characteristics of 1.29- $\mu \mathrm{m}$ GaInNAs ridge-waveguide laser diodes," IEEE Photon. Technol. Lett., vol. 12, no. 6, pp. 597-599, Jun. 2000.

[22] A. Martinez, J. G. Provost, B. Dagens, V. Sallet, D. Jahan, K. Merghem, L. Ferlazzo, J. Landreau, O. Le Gouezigou, J. C. Harmand, and 
A. Ramdane, "9.7 GHz small-signal bandwidth of three-quantum well GalnNAs/GaAs laser diodes operating at $1.35 \mu \mathrm{m}$," Electron. Lett., vol. 40 , pp. 425-427, 2004.

[23] L. Shterengas, J. Y. Yeh, L. Mawst, N. Tansu, and G. Belenky, "Linewidthenhancement factors of InGaAs and InGaAsN single-quantum-well diode lasers," Proc. Conf. Lasers and Electro-Optics (CLEO'04), vol. 1, San Francisco, CA, May 2004.

[24] G. Steinle, F. Mederer, M. Kicherer, R. Michalzik, G. Kristen, A. Y. Egorov, H. Riechert, H. D. Wolf, and K. J. Ebeling, "Data transmission up to $10 \mathrm{Gbit} / \mathrm{s}$ with $1.3 \mu \mathrm{m}$ wavelength InGaAsN VCSELs," Electron. Lett., vol. 37, pp. 803-803, 2001.

[25] S. Tomic, E. P. O’Reilly, R. Fehse, S. J. Sweeney, A. R. Adams, A. D. Andreev, S. A. Choulis, T. J. C. Hosea, and H. Riechert, "Theoretical and experimental analysis of $1.3-\mu \mathrm{m} \mathrm{InGaAsN/GaAs} \mathrm{lasers,"} \mathrm{IEEE}$ J. Sel. Topics Quantum Electron., vol. 9, no. 5, pp. 1228-1238, Sep. 2003.

[26] S. Tomic and E. P. O'Reilly, "Gain characteristics of ideal dilute nitride quantum well lasers," Phys. E, vol. 13, pp. 1102-1105, 2002.

[27] D. Alexandropoulos and M. J. Adams, "Gain, differential gain and linewidth enhancement factor of GaInNAs/GaAs strained quantum well lasers," J. Phys., vol. 14, pp. 3523-3536, 2002.

[28] J. M. Ulloa, J. L. Sanchez-Rojas, A. Hierro, J. M. G. Tijero, and E. Tournie, "Effect of nitrogen on the band structure and material gain of $\operatorname{In}_{\mathrm{y}} \mathrm{Ga}_{1-\mathrm{y}} \mathrm{As}_{1-\mathrm{x}} \mathrm{N}_{\mathrm{x}}$ GaAs quantum wells," IEEE J. Sel. Topics Quantum Electron., vol. 9, no. 3, pp. 716-722, May/Jun. 2003.

[29] R. Nagarajan, T. Fukushima, S. W. Corzine, and J. E. Bowers, "Effects of carrier transport on high-speed quantum-well lasers," Appl. Phys. Lett., vol. 59, pp. 1835-1837, 1991.

[30] C. Silfvenius, G. Landgren, and S. Marcinkevicius, "Carrier transport effects in $1.3 \mu \mathrm{m}$ multiple quantum well InGaAsP laser design," Jpn. J. Appl. Phys., vol. 38, pp. 1227-1229, 1999.

[31] T. Keating, X. Jin, S. L. Chuang, and K. Hess, "Temperature dependence of electrical and optical modulation responses of quantum-well lasers," IEEE J. Quantum Electron., vol. 35, no. 10, pp. 1526-1534, Oct. 1999.

[32] I. Esquivias, S. Weisser, B. Romero, and J. D. Ralston, "Carrier capture and escape times in $\mathrm{In}_{0.35} \mathrm{Ga}_{0.65}$ As-GaAs multiquantum-well lasers determined from high-frequency electrical impedance measurements," IEEE Photon. Technol. Lett., vol. 8, no. 10, pp. 1294-1296, Oct. 1996.

[33] L. A. Coldren and S. W. Corzine, Diode Lasers and Photonic Integrated Circuits. New York: Wiley, 1995.

[34] N. Tansu and L. J. Mawst, "The role of hole leakage in 1300-nm InGaAsN quantum-well lasers," Appl. Phys. Lett., vol. 82, pp. 1500-1502, 2003.

[35] J. J. Liou and F. A. Lindholm, "Capacitance of semiconductor p-n-junction space-charge layers: An overview," Proc. IEEE, vol. 76, no. 11, pp. 14061422, Nov. 1988

[36] B. Sheinman and D. Ritter, "Capacitance of abrupt one-sided heterojunctions," IEEE Trans. Electron Devices, vol. 50, no. 4, pp. 1075-1080, Apr. 2003

[37] O. Anton, C. S. Menoni, J. Y. Yeh, L. J. Mawst, J. M. Pikal, and N. Tansu, "Increased monomolecular recombination in MOCVD grown $1.3-\mu \mathrm{m}$ InGaAsN-GaAsP-GaAs QW lasers from carrier lifetime measurements," IEEE Photon. Technol. Lett., vol. 17, no. 5, pp. 953-955, May 2005.

[38] K. L. Lau, "Dynamics of Quantum Well Lasers," in Quantum Well Lasers, P. Zory, Ed. New York: Academic, 1993

[39] J. M. Pikal, C. S. Menoni, H. Temkin, P. Thiagarajan, and G. Y. Robinson, "Impedance independent optical carrier lifetime measurements in semiconductor lasers," Rev. Sci. Instrum., vol. 69, pp. 4247-4248, 1998.

[40] P. M. Smowton and P. Blood, "Fermi level pinning and differential efficiency in GaInP quantum well laser diodes," Appl. Phys. Lett., vol. 70, pp. 1073-1075, 1997.

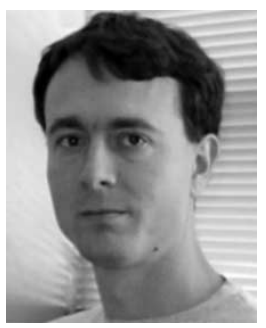

Ovidio H. Anton (S'99) received the B.S. degree in electronics engineering from UNdMdP, Argentina and the M.S. degree in electrical engineering from Colorado State University in 1999 and 2002, respectively. He is currently working towards the Ph.D. degree at Colorado State University.

His research focuses on the investigation of static and dynamic properties of mid-infrared lasers of telecom applications. His combined 15-year experience comprises the design and simulation of electronic circuits plus the experimental skills in optics, lasers and laser software modeling, reflected by 14 peer-reviewed publications and conference proceedings.

Dr. Anton is a member of IEEE/LEOS and Audio Engineering Society. He is the recipient of the Agilent Photonics Fellowship Award at Colorado Sate University.

Dinesh Patel received the B.Sc. and Ph.D. degrees from the University of Surrey, U.K. He is currently a Research Associate at the Electrical and Computer Engineering Department, Colorado State University, Fort Collins.

His present research areas includes characterization of gain in semiconductor laser with temperature and high hydrostatic pressure, and the development of advanced optical coatings using assisted ion beam sputter deposition system. $\mathrm{He}$ also has ongoing efforts in chemically assisted ion-beam etching of nitride and related materials.

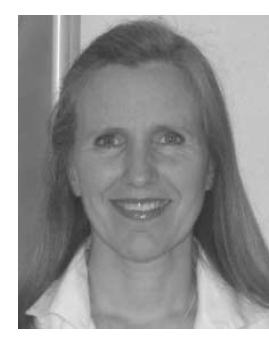

ultraviolet lasers.

Prof. Menoni is a member of the American Physical Society and Optical Society of America. She is Associate Editor of the IEEE JOURNAL OF QUANTUM ELECTRONICS, member of the editorial board of the Review of Scientific Instruments, has Co-Chaired the SPIE Symposium on Semiconductor Lasers of Lightwave Communications at ITCOM 01 and 02, and has been member of the Optical Materials, Fabrication and Characterization Committee, Conference on Lasers and Electro-Optics.

Jeng-Ya Yeh received the B.S. degree in physics from the National Tsing Hua University, Hsinchu, Taiwan, R.O.C. in 1996. He is currently working toward the Ph.D. degree in the Department of Electrical and Computer Engineering at the University of Wisconsin-Madison.

$\mathrm{He}$ is currently a Research Assistant in the Reed Center for Photonics at the University of Wisconsin-Madison. His primary research interest focuses on developing high-performance long wavelength (1300-nm and beyond) InGaAsN QW lasers by metalorganic chemical vapor deposition (MOCVD) for optical communication. His research interests include optimization and physical understanding of the laser characteristics of InGaAsN QW lasers.

T. T. Van Roy, photograph and biography is not available at the time of publication.

L. J. Mawst (M'88-SM'93) was born in Chicago, IL, in 1959. He received the B.S. degree in engineering physics and the M.S. and Ph.D. degrees in electrical engineering from the University of Illinois at Urbana-Champaign in 1982, 1984, and 1987, respectively. His dissertation research involved the development of index-guided semiconductor lasers and laser arrays grown by MOCVD.

He joined TRW, Inc., Redondo Beach, CA, in 1987, where he was a Senior Scientist in the research center, engaged in design and development of semiconductor lasers using MOCVD crystal growth. He is co-inventor of the Resonant Optical Waveguide (ROW) antiguided array and has contributed to its development as a practical source of high coherent power, for which he received the 
TRW Group Level Chairman's award. He developed a novel single-mode edgeemitting laser structure, the ARROW laser, as a source for coupling high powers into fibers. He has also been involved in the development of two-dimensional coherent surface-emitting arrays, vertical-cavity surface emitters, and disributedfeedback laser structures. He is currently a Professor in the Electrical and Computer Engineering Department at the University of Wisconsin-Madison, where he is involved in the development novel III/V compound semiconductor device structures, including vertical cavity surface emitters (VCSELs), active photonic lattice structures, InGaAsN lasers, and high-power Al-free diode lasers. He has authored or coautored more than 140 technical papers and holds 18 patents.

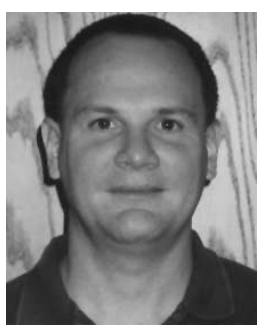

J. M. Pikal received the B.S. degree from Purdue University, the M.S. degree from University of Colorado, and the Ph.D. degree from Colorado State University in 1988, 1993, and 1999 respectively, all in electrical engineering.

$\mathrm{He}$ is currently an Associate Professor of electrical and computer engineering at the University of Wyoming, Laramie, where his research focuses on understanding the properties of semiconductor lasers through studies of carrier lifetime, recombination, and gain.

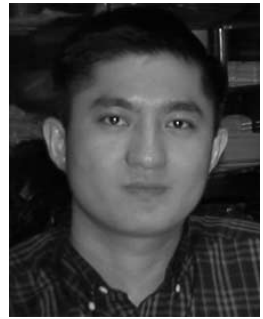

Nelson Tansu was born in Medan, North Sumatra, Indonesia, in October 1977. He received the B.S. degree in applied mathematics, electrical engineering, and physics (AMEP) and the Ph.D. degree in electrical engineering, with distributed minors in physics and material science engineering, from the University of Wisconsin-Madison in 1998 and 2003, respectively.

Since July 2003, he has been an Assistant Professor in the Department of Electrical and Computer Engineering, within the P. C. Rossin College of Engineering and Applied Science at Lehigh University, Bethlehem, PA. He has also been a faculty member in the Center for Optical Technologies at Lehigh University since July 2003. His research works include design, fabrication, MOCVD epitaxy, and device physics of novel-active-material based on semiconductornanostructure optoelectronic devices for all practical transmission windows of optical-communications systems. His focus is also on the physics of semiconductor quantum-well lasers encompassing recombination mechanisms, optical gain, carrier transport, and temperature characteristics. He has published over 77 papers in numerous refereed international journal and conference publications. He also currently holds several U.S. patents in the fields of semiconductor optoelectronics devices and high power semiconductor lasers. He has given numerous lectures, seminars, and invited talks in universities, research institutions, and conferences worldwide.

Prof. Tansu was a recipient of the Bohn Scholarship, the WARF Graduate University Fellowship, the Vilas Graduate University Fellowship, and the Graduate Dissertator Travel Funding Award at the University of Wisconsin-Madison. He also received the 2003 Harold A. Peterson ECE Best Research Paper Award (1st Prize) from the University of Wisconsin-Madison. 